\title{
Réclamer Viva Voce
}

\section{Charles Tilly}

\section{OpenEdition \\ 1 Journals}

\section{Édition électronique}

URL : http://journals.openedition.org/conflits/143

DOI : $10.4000 /$ conflits. 143

ISSN : 1777-5345

Éditeur :

CCLS - Centre d'études sur les conflits lilberté et sécurité, L'Harmattan

Édition imprimée

Date de publication : 21 mai 1992

ISSN : 1157-996X

Référence électronique

Charles Tilly, « Réclamer Viva Voce », Cultures \& Conflits [En ligne], 05 | printemps 1992, mis en ligne le 07 janvier 2003, consulté le 30 mars 2021. URL : http://journals.openedition.org/conflits/143 ; DOI https://doi.org/10.4000/conflits. 143

Ce document a été généré automatiquement le 30 mars 2021.

Creative Commons License 


\title{
Réclamer Viva Voce
}

\author{
Charles Tilly
}

1 L'année 1989 a donné du travail aux observateurs des mouvements sociaux en Europe. Ceux qui pensaient jusqu'ici pouvoir se contenter d'établir des parallèles entre les mouvements existant dans les différents pays se trouvèrent obligé d'expliquer non seulement l'existence de liens entre des mouvements dispersés mais également les rapports existant entre les contextes politiques de ces différents mouvements. Alors que la précédente vague de revendications qui avait touché l'Europe - celle de 1968 était restée circonscrite à la partie occidentale du continent, pour celle-ci l'initiative est venue directement de l'Est, URSS comprise, bien que ce pays soit considéré depuis longtemps comme un cas à part. Dans les pays baltes, en Tchécoslovaquie, en Roumanie, voire en Albanie, des citoyens sont descendus dans la rue pour crier "viva voce" des doléances qu'ils n'exprimaient jusqu'alors que dans les cercles de café ou par des samizdats.

Le réseau des événements de 1989

2 Après son arrivée au pouvoir en 1985, Mikhaïl Gorbatchev a annoncé clairement ses intentions de réduire les dépenses militaires, d'améliorer les relations de l'URSS avec les Etats-Unis et l'OTAN, et de freiner l'intervention militaire soviétique à l'étranger. L'échec, coûteux et traumatisant, d'Afghanistan - le face-à-face le plus sérieux avec le pouvoir militaire américain depuis plusieurs années - a considérablement entamé le prestige de l'armée soviétique, faisant planer des doutes sur la politique de parité militaire avec les USA. Le programme gorbatchévien de non-ingérence et de démilitarisation s'est même étendu aux pays satellites de l'Europe de l'Est.

3 Lorsque l'URSS eut entamé la réduction de ses dépenses militaires et annoncé son retrait à l'étranger, les citoyens des autres pays de l'Est ont commencé à penser que la probabilité d'un soutien militaire soviétique à leur gouvernement en cas de défis internes baissait. En URSS même, les habitants des régions non russes ont tiré des conclusions similaires. L'assouplissement du caractère répressif du régime a encouragé l'émergence de revendications depuis longtemps silencieuses.

4 Dès lors, les défis se sont multipliés en Pologne, Hongrie, Tchécoslovaquie, et en Allemagne de l'Est. Partout, sauf en Allemagne, des forces d'opposition politique 
s'étaient constituées depuis au moins dix ans. Le "dégel" de 1985 permit leur essor. En juin 1989, les Polonais désignèrent 99 candidats anticommunistes sur les 100 qui se présentèrent à la chambre supérieure au cours d'élections "plutôt" libres. A la chambre inférieure, la loi électorale limitait Solidarité à $35 \%$ des sièges, mais le Parti paysan avait rompu en 1989 son alliance permanente avec les communistes, donnant ainsi à Solidarité une majorité et au pays un premier ministre non communiste: Tadeusz Mazowiecki. Les Soviétiques sont restés silencieux devant ces changements. Un renversement du pouvoir communiste s'est également produit en Hongrie, où le Parti socialiste ouvrier s'est "suicidé" avant la fin de l'année pendant qu'un référendum national imposait le démantèlement des cellules communistes d'usine, la dissolution des milices communistes et la publication des comptes du feu Parti. De leur côté, les gouvernants tchécoslovaques ont réussi à interdire les manifestations jusqu'en novembre 1989, mais la fuite des réfugiés est-allemands à travers le pays, la condamnation rétrospective de l'invasion de la Tchécoslovaquie en 1968 par deux pays intervenants (la Hongrie et la Pologne), et la transformation subite des voisins excommunistes ont accentué la pression exercée sur le régime. A la mi-novembre, des manifestations de masse à Prague et dans tout le pays arrêtèrent la machine gouvernementale, accélérant la formation d'une opposition publique: le Forum civique, et provoquant une série d'improvisations qui amenèrent Dubcek et Havel au pouvoir.

Les Allemands de l'Est, à la différence de leurs voisins, avaient manifesté une résistance plutôt "molle" au régime communiste avant l'effondrement de 1989. Cependant au cours de l'automne 1989, les autorités tchèques, polonaises et hongroises permirent à des milliers d'est-allemands de passer leurs frontières pour aller à l'Ouest, la nonintervention des forces soviétiques et l'incapacité du régime est-allemand à arrêter cette fuite constituèrent alors deux indices révélateurs des transformations qui étaient en train de s'opérer. Les Allemands de l'Est commencèrent alors à manifester pour une réforme démocratique et contre leurs médiocres conditions de vie. Lorsque les leaders de l'opposition de toute l'Europe de l'Est apprirent que l'ambassade soviétique de Prague avait écouté calmement une délégation du Forum Civique au lieu de la repousser, ils en tirèrent les conséquences. Bientôt des révoltes populaires, très diverses, balayèrent les chefs communistes de Roumanie, de Bulgarie et d'Albanie. Il faudrait ajouter à cela la multiplication des revendications d'indépendance ou d'autonomie à l'intérieur de la Yougoslavie, de la Tchécoslovaquie, et même de l'Union soviétique. L'URSS a alors entamé ce processus de désagrégation qui a abouti à sa disparition à la fin de 1991.

Bien que chaque mouvement national présente des particularités inhérentes à sa propre histoire politique, on peut sans nul doute affirmer que les manifestations, les cortèges, les meetings qui se déroulèrent un peu partout en Europe de l'Est en 1989 ont puisé dans un stock commun de connaissances et de références. Il ne s'agit pas cependant d'une série d'actions parallèles qui auraient subi des influences identiques, mais d'un réseau d'événements qui dépendent étroitement les uns des autres. Ceci nous amène à poser immédiatement une question d'ordre théorique : Comment concevoir les rapports existant entre des mouvements simultanés, mais dispersés géographiquement, afin de comprendre la manière dont cette "libération" a pu se répandre aussi rapidement et aussi largement? Il ne s'agira cependant point ici de suivre le déroulement au jour le jour des mouvements de 1989, ou d'exposer un modèle 
explicatif, mais plutôt de présenter une esquisse des perspectives envisageables permettant au demeurant de comprendre ce qui s'est passé.

Les problématiques disponibles

7 A l'image de ce qui s'est passé il y a un quart de siècle, les événements de 1989 susciteront certainement de nouvelles réflexions sur l'action collective au fur et à mesure de la prise de conscience des faiblesses des modèles explicatifs actuels qui deviennent évidentes quand on tente de les utiliser pour rendre compte de la montée subite des revendications d'indépendance dans toute l'Europe de l'Est. Différentes modélisations de la mobilisation politique et de l'action collective, dont les miennes, ont été élaborées à partir des mouvements des années 60 en Europe et en Amérique. Elles mettaient en cause tout d'abord les notions de "comportement collectif" non rationnel qui avaient dominé jusqu'alors, à la fois l'analyse savante et le discours officiel sur les émeutes, les révolutions, et les mouvements sociaux - courant qui trouvait son expression la plus élaborée dans les travaux de Neil Smelser, Ralph Turner et Chalmers Johnson. En réponse à cette tradition, les modélisations de l'action collective populaire ayant pour interprètes et défenseurs Francesco Alberoni, William Gamson et George Rudé accentuèrent au contraire la rationalité, la solidité, l'organisation, la conscience calculée, voire l'efficacité des mouvements que d'autres dénommaient spontanés voire irrationnels. Le deuxième aspect de la critique porta sur l'idée marxisante d'une marche générale de l'histoire au cours de laquelle les groupes (et surtout en fait les classes sociales) prennent conscience d'eux-mêmes, en formant des mouvements sociaux ou, mieux, en se joignant au mouvement social général, à la Sozialbewegung qui donne un sens profond à l'histoire. Alors que plusieurs des modèles de comportements collectifs évoquaient l'existence d'un lien plus ou moins direct et automatique entre intérêt matériel et action collective, Mancur Olson précisa que le passage entre intérêt et action, loin d'être libre, était au contraire semé d'embûches, et notamment celles laissées par les free riders. Cette école olsonienne assimilant l'analyse de l'action collective à celle de la production de biens collectifs, donna au sujet un parfum d'économisme.

8 De cette double remise en cause des idées reçues est né un ensemble d'idées parfois contradictoires, pas toujours cohérentes, et souvent incomplètes qui partent de l'idée que l'action collective est incertaine, coûteuse, délibérée, issue d'un jeu entre intérêt, organisation et conscience. Même les travaux postérieurs sur les nouveaux mouvements sociaux, notamment ceux d'Alain Touraine ou d'Alberto Melucci, qui insistent sur l'importance de l'identité partagée et rejètent la canalisation de toute action collective par l'état, acceptent en général cette manière de poser le problème. La nouvelle problématique présente plusieurs avantages sur les précédentes, mais elle suppose pour ainsi dire une analyse "basiste" ou "monadique" puisqu'elle part toujours de l'origine populaire des mouvements en postulant l'existence d'acteurs plus ou moins isolés dont la situation et le caractère peuvent expliquer l'action. Dans cette optique, on élabore alors des explications acteur par acteur, groupe par groupe, en acceptant sans beaucoup y réfléchir que l'objet de l'explication soit nécessairement le comportement de ceux qui protestent. Or, cette façon de poser le problème occulte à la fois les comportements des dominants et le jeu d'interaction entre acteurs. On voit donc à quel point une telle problématique constitue un handicap sérieux à toute analyse des événements de 1989 en Europe de l'Est. 
9 Pour résumer, les modèles explicatifs dominants de l'action collective ont beaucoup évolué depuis les années 60 . Les premiers modèles - y compris les miens - paraissent maintenant naïfs avec leur rationalisme simpliste, leur absence de référence au contexte social, leur présupposition portant notamment sur des acteurs déjà constitués, dont la position politique reste plus ou moins stable et fixe. L'élaboration d'une conception plus historique de l'évolution des formes de l'action collective, l'approfondissement de l'étude des rapports entre l'organisation de la vie quotidienne et la participation aux conflits, et enfin la conceptualisation des acteurs comme réseaux sociaux changeants, contingents et construits, sujets eux-mêmes à des processus politiques et idéologiques, sont à l'origine des profondes modifications enregistrées par les modèles qui étaient proposés à l'époque.

Apports et limites des modélisations actuelles

On arrive aujourd'hui à une bifurcation partielle du champ des analyses: on trouve d'un côté, les analyses complexes de l'action rationnelle qui reposent sur la présomption d'acteurs et d'intérêts donnés a priori, et de l'autre une gamme d'études historicistes qui rattachent étroitement l'action collective à des transFormations politiques, économiques et idéologiques. Toutefois à l'heure actuelle, même les approches très rationalistes, comme celle de Michael Hechter, cherchent à établir les rapports existant entre l'action collective et le contexte historique. On ne saurait certes parler en la matière de nouvelle synthèse qui déboucherait sur un consensus, mais on peut cependant affirmer qu'il y a accord sur la nécessité de lier les processus historiques, économiques, politiques, idéologiques, et ceux de l'action collective.

11 Les études sur la révolution et sur l'état ont suivi une trajectoire parallèle, sinon convergente. Dans les études portant sur les périodes révolutionnaires, les modèles d'histoire naturelle à la Crane Brinton ont disparu, remplacées par de nombreuses analyses comparatives à l'image de celle proposée par Theda Skocpol.

12 L'analyse des révolutions a été rattachée alors à celle de la transformation des états, ce qui constituait dans une certaine mesure une grande avancée théorique. Côté état, on assiste actuellement à une disparition progressive des conceptions typologiques et évolutionnistes au profit d'une démarche plus réaliste qui distingue l'état de son environnement social, afin précisément de cerner les liens (conçus maintenant comme contingents et même comme précaires "contrairement" aux conceptions proposées par Althusser) existant entre eux. Ainsi, les travaux de Michael Mann, par exemple, présentent une image de l'état, structure complexe où se croisent les fils du pouvoir social, mais qui agit cependant de manière puissante sur la vie sociale.

13 Les représentations des mouvements sociaux ont subi une évolution semblable. Nous entendons par "mouvement social" le fait d'organiser des revendications à l'encontre des autorités nationales au nom de populations lésées et sur la base d'associations intéressées - donc une forme d'action politique qui naquit (ou du moins se diffusa largement) en Europe au XIXème siècle pour devenir au XXème totalement banale. On concevait encore dans les années 60 le mouvement social (mouvement pour le suffrage féminin, lutte contre l'alcoolisme, etc.) comme une espèce de baleine qui surgit hors de l'eau, disparaît puis réapparaît tout en restant identique à elle-même, ce qui permet d'en suivre l'itinéraire aquatique. Les études générales sur la mobilisation politique, les analyses de la participation par le biais de l'interview, par observation directe et par la consultation des archives policières, ainsi que l'histoire sociale des transformations politiques ont par la suite elles-mêmes été l'objet d'une historicisation du sujet. On a 
effectué notamment une distinction entre mouvement-action et mouvementorganisation, noté l'existence d'un personnel flottant dans presque tout mouvement durable, suivi les conflits et les coalitions à l'intérieur de la plupart des mouvements, et enfin insisté sur l'interaction stratégique ininterrompue entre organisations, réseaux de base, concurrents et diverses autorités. ces mutations ont contribué en définitive à supprimer l'image du mouvement social comme groupe cohérent et continu, image qui vient d'ordinaire de la façon dont les mouvements se pensent et de la manière dont leurs dirigeants cherchent à établir l'authenticité de leur leadership à travers l'image des liens qu'ils entretiennent avec la base.

Bien que les définitions proposées restent controversées, les chercheurs semblent maintenant en accord avec une conception des mouvements sociaux entendus comme forme d'action politique qui repose sur une vaste gamme d'organisations et de réseaux où l'association spécialisée occupe depuis 150 ans une place centrale, mais non unique. On accepte en général l'idée que depuis 1945 de nombreuses associations se sont formées, en Amérique et en Europe de l'Ouest, pour lier les actions et la clientèle des mouvements sociaux aux astuces et aux permanences des lobbies. Les spécialistes se divisent plus nettement sur la question de savoir si le mouvement social a été une invention politique de l'époque de la Révolution française qui s'est diffusé à travers l'Ouest ou s'il s'agit d'un phénomène beaucoup plus général et ancien qui présente différents aspects selon la conjoncture historique. Cependant personne ne conteste l'idée d'une prolifération des mouvement sociaux depuis 1789.

15 Les spécialistes se battent également au sujet des "nouveaux mouvements sociaux" comme les mouvements écologiste et féministe. Les uns prétendent que ces mouvements franchissent les limites posées par leurs prédécesseurs en cherchant des transformations fondamentales de la vie plutôt que les avantages ordinaires d'un pouvoir accru, les autres soulignent les continuités dans l'organisation et la stratégie. Heureusement, les nombreuses analyses empiriques actuelles qu'a suscité cette controverse promettent de nous fournir une réponse au moins partielle à la question. Les résultats me semble-t-il, confirmeront une certaine continuité entre soi-disant anciens et soi-disant nouveaux mouvements sociaux, mais déplaceront l'enquête vers les relations réciproques entre mouvement, environnement politique, et milieux de participation.

La notion de cycle de contestation

16 A partir de ses études des conflits italiens entre 1965 et 1975, Sidney Tarrow a renouvelé la discussion en proposant sa conception des cycles de contestation. Les revendications réussies ont tendance, selon Tarrow, à susciter d'autres revendications de la part d'acteurs différents, certains d'entre eux apercevant une ouverture jusqu'ici invisible ou inaccessible et d'autres au contraire ressentant une menace sur des positions acquises. L'expansion se poursuit jusqu'au "moment" (phase) où les concurrents se fixent, s'établissent, s'essoufflent, s'entre-tuent ou succombent à la répression étatique diligentée par ceux qui se sentent menacés par la complaisance des autorités.

17 Au cours d'un tel cycle, les premières phases bourgeonnent d'innovations, créent aux marges des revendications un espace où se déploient différentes expériences collectives, ce qui donne l'impression d'une rupture totale avec le passé. Chaque grand cycle laisse, en effet, ses traces sur le système politique: formation de nouveaux groupements, réaménagements des rapports avec les pouvoirs publics, rénovation du 
discours politique, établissement de nouveaux moyens d'actions. Toutefois, ces résultats restent à la fois en deçà des espoirs et des craintes formulés lors de l'apogée de la contestation, mais décevant nombre de ceux qui sont descendus dans la rue, notamment les membres des groupes qui arrivent en bout de cycle sans gratification et qui n'ont pour se consoler que le sentiment d'avoir préservé leur pureté, ce qui n'est pas le cas des leaders "vendus" qui ont gagné quelque chose. Cet ensemble de conséquences promeut la doctrine de la rupture, qui après le dernier cycle européen (celui de 1965-1975 environ) a donné justement naissance à l'idée de nouveaux mouvements sociaux.

Le schéma de Tarrow se base sur une conception de la structure des opportunités politiques $^{1}$ (political opportunity structure) contraignante pour tous les acteurs, variant d'un pays à l'autre et d'une année sur l'autre. En principe l'action collective pourrait subir des mutations brusques sans modifications substantielles du caractère ou de la situation immédiate des acteurs, pourvu seulement que la structure des opportunités change rapidement (par exemple, au début d'une guerre internationale ou à la mort d'un roi). Le principe demeure cependant théorique. Dans la pratique de telles mutations provoquent toujours simultanément des processus de mobilisation, de démobilisation, de formation, de coalescence, de désintégration et de dissolution des acteurs collectifs possibles. Mais le concept conserve toutefois sa pertinence indépendamment de cette remarque, car il aide à identifier les parallèles - qui sinon sont difficiles à saisir - existant entre les cycles de revendications et d'autres phénomènes politiques comme les situations révolutionnaires, les fins de guerres, et la désintégration d'empires. Une situation révolutionnaire se forme lorsqu'au moins deux blocs à l'intérieur du même état prétendent gérer exclusivement les mêmes instruments étatiques en recevant chacun le soutien d'une part importante des citoyens de cet état. Une situation révolutionnaire n'aboutit pas nécessairement à une révolution accomplie, puisqu'un bloc représentant l'ancien régime peut supprimer son rival, ou l'état peut se fractionner en permanence. En revanche, une révolution "pleine" - c'est-à-dire un transfert important de pouvoir étatique à travers une lutte violente - ne s'opère pas sans situation révolutionnaire. Une situation révolutionnaire peut débuter avec la déclaration d'indépendance d'un segment subordonné d'un état (par exemple l'Estonie à l'intérieur de l'état soviétique de 1990), par la mobilisation d'une coalition d'acteurs auparavant exclus, en partie au moins, du pouvoir (par exemple les coalitions d'intellectuels, de bourgeois, et d'artisans qui se formèrent dans plusieurs pays au cours de l'année 1848) ou par la scission en blocs d'une coalition auparavant dominante (par exemple, le clivage entre Roundheads et Cavaliers en Angleterre après 1640).

19 Les situations révolutionnaires poussent à l'extrême une condition politique qui se présente aussi hors des révolutions, à savoir un glissement du pouvoir étatique qui menace tous ceux qui gardent un intérêt à la distribution présente du pouvoir, mais qui ouvre cependant en même temps des opportunités à tous les autres acteurs - y compris ceux des classes dominantes - ayant la capacité de faire jouer leurs intérêts par une action rapide et décisive.

20 Par exemple, même lorsqu'elles ne se traduisent pas par un clivage à l'intérieur d'un état, les fins de guerre présentent souvent cette caractéristique. Lors d'une grande mobilisation militaire, presque tous les états promettent plus qu'ils ne peuvent offrir en temps de paix. Ces promesses qui entraînent l'augmentation de la dette publique 
prennent la forme d'avantages sociaux pour les ouvriers, les fonctionnaires, les sinistrés de guerre (prise en charge pour les d'avantages financiers anciens combattants et leurs familles, etc.), pour les capitalistes, de privilèges pour les minorités ethniques qui en échange arrêtent leurs revendications pendant l'état d'urgence. De plus, les états érigent souvent au cours d'une guerre des contrôles extraordinaires sur la vie économique, politique et sociale qu'ils démantèlent à la fin des hostilités, au moment où ils démobilisent la production militaire et réintègrent les soldats à l'économie civile. Cette détente favorise un déferlement de réclamations différées depuis longtemps qui peuvent déborder subitement la capacité de l'état. Plus l'état a perdu de capacité et de crédibilité pendant la guerre - défaite et occupation étrangère étant les conditions limites - plus les risques de débordement sont grands.

21 Ces conditions menacent les droits établis et rendent l'état vulnérable à de nouvelles revendications. Prenons pour exemple la situation à la fin de la Première Guerre mondiale. Tous les états engagés dans le combat, y compris les Pays-Bas soi-disant neutres et les Etats-Unis entrés tardivement dans le conflit, ont été confrontés aux défis lancés par des acteurs politiques qui avaient participé activement aux préparatifs de la guerre. Le poids du défi lancé aux états s'est alourdi devant l'étendue des dommages de guerre. Seules la Russie et l'Allemagne, ravagées par la guerre, ont connu de véritables situations révolutionnaires. L'Italie, avec ses grèves géantes, ses occupations d'usines, et son immense mobilisation fasciste, a connu une situation politique proche d'une situation révolutionnaire. La France, la Grande-Bretagne et les Etats-Unis (dans cet ordre), ont également, au même moment, dû faire face à des vagues de revendications, de moindres importances cependant. La désintégration des empires, des fédérations, et des coalitions présente des caractéristiques comparables à celles des situations révolutionnaires. La sécession - sans sanction - d'un membre visible transmet toute une série de renseignements et de signaux aux autres membres: la diminution de la capacité de l'autorité centrale à la fois à remplir ses obligations et à renforcer les obligations des autres, l'augmentation probable des coûts d'une fidélité au centre, et donc en définitive la prise de conscience de la possibilité de la sécession et de l'intérêt d'une collaboration avec les premiers sécessionnistes. Mikhaïl Gorbatchev a goûté à cette logique amère, après la sécession de l'Estonie, de la Lettonie et de la Lituanie. Ces différents processus politiques se déroulent donc selon des principes qui s'apparentent à ceux des vagues de mouvements sociaux. Un ensemble de revendications favorise l'émergence d'autres revendications, la concurrence et la lutte pour la détention du pouvoir politique entre les organisations s'accentuent, les revendications se multiplient, se généralisent avant de se dissiper. Au cours du processus, des militants inventent et testent de nouvelles façons d'organiser et de s'organiser, d'articuler leurs buts, de revendiquer, de combattre leurs ennemis, de discipliner leurs adhérents, de protéger les avantages déjà acquis. A la fin du cycle, certains acteurs ont obtenu une partie du pouvoir, d'autres ont vu leurs ressources politiques rognées, le discours public sur les enjeux s'est transformé, tout au moins partiellement, et l'ensemble des moyens acceptables d'action collective, c'est-à-dire le répertoire de contestation, a subi également des modifications.

$\mathrm{Au}$ cours de ces cycles, les revendications initiales présentent deux caractéristiques. Elles tendent à imposer au pouvoir la prise en considération des dispositions qu'elles proposent, ce qui offre à tout acteur politique une occasion immédiate d'agir. Elles menacent ensuite inévitablement les intérêts des acteurs soit parce que la redistribution des bénéfices diminuera les ressources disponibles et accessibles aux 
acteurs restants, soit parce que les revendications s'attaquent directement aux intérêts d'un groupe établi. Les situations révolutionnaires et non révolutionnaires présentent de tout évidence des aspects parallèles. En effet, la multiplication des situations révolutionnaires et quasi révolutionnaires en Europe entre 1847 et 1849, partage nombre de caractéristiques communes avec les cycles de protestations plus restreints. La vulnérabilité d'états importants au programme révolutionnaire (tels les états belge et napolitain avant la révolution française de 1848) rend plus probable l'émergence de tels programmes dans d'autres pays, favorise les prédispositions et la disponibilité des acteurs à la doctrine et à l'expertise révolutionnaires, et réduit la propension des états qui s'engagent eux-mêmes dans la voie révolutionnaire à intervenir en faveur des anciens régimes voisins.

La communication et les croyances partagées jouent un rôle critique au cours de ces processus révolutionnaires et quasi-révolutionnaires. Trois éléments sont amenés à se transformer au cours de ces processus: premièrement, la capacité de l'état; deuxièmement, les croyances partagées parmi les acteurs politiques quant aux conséquences probables des diverses revendications et actions y compris les croyances au sujet de la capacité de l'état; troisièmement, les capacités relatives des divers acteurs d'agir collectivement, qui comprennent d'une part la formation de nouveaux acteurs par création, mobilisation, ou coalition, et d'autre part, la diffusion des doctrines des connaissances techniques, et des militants.

Désastres naturels et invasion militaire mis à part, la capacité des états évolue en général lentement. La rapidité du passage entre situation non-révolutionnaire et situation révolutionnaire, situation de passivité et situation d'activité, dépend en général non des bouleversements dans la vulnérabilité des états, mais de la diffusion rapide de nouvelles informations, de nouvelles croyances, de nouvelles évaluations des conséquences probables de l'action.

Retour sur les événements de 1989 : esquisse de perspectives

On est tenté de croire que le rôle de la télévision et de la presse explique l'internationalisation des formes et des rythmes de l'action collective tant les manifestations et les révoltes lointaines bénéficient d'une publicité de plus en plus immédiate et importante de la part des médias, quand les actions ne sont pas tout simplement fabriquées ou déformées pour et par les médias. Ce serait cependant aller un peu trop vite que de s'en tenir à cette seule explication et de confondre médiatisation avec internationalisation. Faut-il rappeler tout d'abord la portée extraordinaire des révolutions de 1789 et 1848 en Europe pour comprendre combien les milieux politiques du continent se sont sentis liés les uns aux autres bien avant la venue de la télévision et du journalisme de masse. Les radicaux et les conservateurs anglais de 1789 ont suivi pratiquement au jour le jour les événements de Paris, et la construction de barricades "à la parisienne" était devenue pratique courante dans l'Allemagne de 1848. Devons-nous faire remarquer ensuite que ce ne sont pas tant les intérêts partagés, la conscience de l'oppression ou les modèles d'actions collectives qui franchissent rapidement les frontières internationales que les informations à l'égard de la vulnérabilité des autorités, de la probabilité d'un soutien international, et de l'efficacité courante de diverses formes d'action. Doit-on conclure enfin, que si une internationalisation des conflits a lieu, elle résulte surtout et avant tout d'une interdépendance des circonstances politiques d'acteurs situés dans des pays différents? 

dans des pays différents résident tout d'abord dans des processus subis simultanément par les pays (la fin d'une guerre générale, par exemple), puis dans les effets des luttes politiques sur les parties tierces, surtout en ce qui concerne les états (par exemple, une révolution rompt l'alliance de deux états, ce qui rend le deuxième état vulnérable à une révolution), enfin dans les emprunts directs, de modèles d'action collective d'informations sur les conséquences (domestiques et internationales) de l'action collective, de croyances à l'égard des buts de l'action, et d'un savoir ou d'une capacité d'expertise des acteurs de l'action collective.

Tous ces liens dépendent étroitement de l'histoire des relations entre les pays en question. Dans le cas de l'Europe de l'Est en 1989 par exemple, le fait d'avoir subi l'hégémonie et parfois l'intervention militaire soviétique, a forgé entre la Pologne, la République démocratique allemande, la Tchécoslovaquie et la Hongrie, des réseaux de communication qui ont renforcé l'extrême sensibilité de ces pays aux changements de relations intervenant entre chacun d'entre eux et l'URSS. L'absence de "réaction" des Soviétiques à la fuite des Allemands de l'Est vers l'Ouest en 1989 a rapidement modifié à la baisse les estimations de la probabilité d'une intervention militaire soviétique dans cette zone. Cette assurance accrue a permis aux Tchèques de porter à la tête de leur nouveau régime la victime de l'intervention soviétique de 1968, Alexandre Dubcek, et un prisonnier de l'ancien régime, récemment libéré, Vaclav Havel. La structure politique antérieure, même dans les moments de grandes turbulences, a donc, sans aucun doute, canalisé la contestation. aboutissent aux mêmes enseignements. En effet, ils établissent nettement l'existence d'une correspondance entre la structure de l'état et l'organisation des militants, comme le montre la dispersion plus grande des associations de base dans les pays fédéraux (Suisse et Allemagne) que dans les pays où l'état est plus centralisé (France et Pays-Bas). Les groupements qui se forment pour contester le pouvoir étatique en empruntent donc les linéaments.

L'histoire intervient encore à un autre niveau. Avec l'accumulation d'études monographiques sur l'action collective, on a commencé, il y a maintenant vingt ans, à établir un lien entre deux faits à savoir: premièrement que les moyens d'action populaire ont changé depuis l'époque du charivari et de l'émeute de subsistances pour se transformer en manifestation et en grande réunion politique; deuxièmement que la gamme des moyens d'action collective réellement disponibles par la population à un moment donné est très limitée par rapport aux possibilités théoriques de l'action collective. On a pris l'habitude de décrire cette situation en termes de répertoires d'action pour accentuer à la fois la contrainte, l'apprentissage et une innovation qui se place la plupart du temps aux marges du répertoire existant plutôt qu'à l'intérieur. Le répertoire de contestation appartient non pas à l'individu ou au groupe, mais aux ensembles d'acteurs politiques - par exemple, les manifestants, les contre-manifestants et la police - qui forment et reforment leur répertoire en répétant leur interaction.

Sidney Tarrow a remarqué récemment combien les répertoires du XVIIIème siècle et des siècles précédents étaient intimement liés aux caractéristiques, aux particularités des réseaux sociaux et des enjeux (l'attaque concertée de la maison d'un transgresseur des règles morales) tandis que les grèves, les manifestations, les réunions publiques, et la plupart des autres formes plus récentes sont plus "modulaires", c'est-à-dire qu'elles

Cultures \& Conflits, 05 | printemps 1992 
se déplacent facilement de groupe en groupe, de pays en pays, de lutte en lutte. Cela explique en partie la généralisation plus rapide des mouvements de nos jours. C'est donc à travers un concept foncièrement historique et culturel qui renforce le sens d'une action, ou plutôt d'une interaction, canalisée non seulement par les structures politiques mais aussi par les connaissances, les croyances, les lignes de communication, que l'explication des mouvements en Europe de l'Est et de l'Ouest, souvent très liés entre eux, pourra se faire. Cela exigera cependant des concepts encore plus historiques et plus interactifs que ceux dont disposent aujourd'hui les chercheurs.

1. Nous avons préféré conserver le terme de "structure des opportunités politiques" plutôt que de recourir à la formule : structure des possibilités politiques. D'une part, parce que le sigle anglais POS est entré dans la littérature consacrée, et, d'autre part, pour insister sur la dimension contextuelle.

\section{Bibliographie}

Alberoni F., Statu nascenti, Bologne : I1 Mulino, 1968.

Anderson B., Imagined Communities. Reflections on the Origin and Spread of Nationalism, London : verso, 1991, 2 éd.

Aya R., Rethinhing Revolutions and Collective Violence. Studies on Concept, Theory, nnd Method, Amsterdam : Het Spinhuis, 1990.

Aymes J.-R., A. Gil Novales \& L. de Oliveira Ramos. Les Révolutions dans le monde ibérique (1766-1834). I. La péninsule, Bordeaux: Presses Universitaires de Bordeaux, 1989.

Baechler J., Les phénomènes révolutionnaires, Paris : Presses Universitaires de France, 1970.

Birbaum P, La logique de l'état, Paris: Fayard, 1982. States and Collective Action: The European Experience, Cambridge : Cambridge University Press, 1988.

Boswell T., (sous la direction de). Revolution in the World System, New York: Greenwood, 1989.

Bright C. \& S. Harding (sous la direction de). Statemahing and Social Movements, Ann Arbor : University ofMichigan Press, 1984.

Brinton C., The anatomy of Revolution, New York : Norton, 1938.

Burke E., (sous la direction de). Global Crises and Social Movements. Artisans, Peasants, Populists, and the World Economy, Boulder, Colorado : Westview, 1988.

Defronzo J., Revolutions and Revolutionary Movemenfs. Boulder Westview, 1991.

Diani M., Isole nell'archipelago. Il movimento ecologista in Italia. Bologna : Il Mulino, 1988. "The Network Structure of the Italian Ecology Movement", Social Science Information 29 (1990) : 5-31.

Dickinson H., "Counter-revolution in Britain in the 1790s", Tijdschrift voor Geschiedenis 102 (1989) : 354-367.

Durandin C., Révolution à la Française ou à la Russe. Polonais Roumains, et Russes au XIXème siècle. Paris : Presses Universitaires de France, 1989.

Favre P, (sous la direction de),La manifestation, Paris: Presses de la Fondation Nationale des Sciences Politiques, 1990. 
Gamson W., The Strategy of Social Protest, Belmont, California : Wadsworth, 1990, 2e éd.

Giugni M., \& H. Kriesi, "Nouveaux mouvements sociaux dans les années 80 : Evolution et perspectives", Annuaire suisse de science politique 30 (1990) : 79-100.

Goldstone J., Revolution and Rebellion in the Early modern World, Berkeley : University of California Press, 1991.

Goodwin J., \& T. Skocpol, "Explaining Revolutions in the Contemporary Third World", Politics and Society 17 (1989), 489-509.

Gould R., Multiple Networks and Mobilization in the Paris Commune, 1871, "American Sociological Review 56 (1991) 729.

Haimson L. \& C. Tilly, (sous la direction de), Strikes, Wars, and Revolutions in an International Perspective. Strike Waves in the Late Nineteenth and Early Twentieth Centuries. Cambridge : Cambridge University Press, 1989.

Hechter M., Principles of Group Solidarity, Berkeley: University ofCalifornia Press, 1987.

Johnson C., Revolutionary Change, Boston : Little, Brown, 1966. Kim Q. (sous la direction de), Revolutions in the Third World Leiden : Brill, 1991.

Kimmel M., Revolution. A Sociological Interpretation, Philadelphia : Temple University Press, 1990.

Klandermans B., H. Kriesi \& S. Tarrow, (sous la direction de). From Structure to Action : Cornparing Social Movement Research Across Cultures. Greenwich, Connecticut : JAI Press, 1988, International Social Movement Research, vol. I.

Klima A., "The Bourgeois Revolution of 1848-9 in Central Europe", in R. Porter \& M. Teich (sous la direction de), Revolution in History Cambridge : Cambridge University Press, 1986. Kossmann E.H., "Liep de Nederlandse Patriottenbeweging op de Franse vooruit ?" in J. Craeybeckx \& F. Scheelings (sous la direction de), De Franse Revolutie en Vlaanderen, Brussels : VUB Press, 1990.

60 Kriesi H., et al. Politische Ahtivierung in der Schweiz, 1945-1978, Diessenhofen : Verlag Ruegger, 1981.

61 Mayer M., "Social Movement Research and Social Movement Practice: The U.S. Pattern", in D. Rucht (sous la direction de), Research on Social Movements : The State of the Art in Western Europe nnd the USA. Frankfurt \& boulder : Campus/Westview, 1991.

McPhail C., The Myth of the Madding Crowd. New York : Aldine De Gruyter, 1991.

Melucci A., Nomads ofthe Present. Social Movements and Individual Need in Contemporary Society, Philadelphia University Press, 1989.

64 Olson M., The Logic of Collective Action. Cambridge, Mass. Harvard University Press, 1965.

Opp K.-D., "Spontaneous Revolutions. The Case of East Germany in 1989", in Heinz Kurz (sous la direction de), 1992. German Unification and European Integration. London : Edward Elgar, 1992.

Outram Dorinda, "Revolution and Repression", Comparative Studies in Society and History 34 (1992) : 58-67. 

New York: Vintage Books, 1979. "Collective Protest: A Critique of Resource Mobilization Theory", International Journal of Politics, Culture and Society 4 (1991) 435-458.

\section{NOTES}

1. Nous avons préféré conserver le terme de "structure des opportunités politiques" plutôt que de recourir à la formule : structure des possibilités politiques. D'une part, parce que le sigle anglais POS est entré dans la littérature consacrée, et, d'autre part, pour insister sur la dimension contextuelle. 


\section{RÉSUMÉS}

Charles Tilly, à propos des événements de 1989 en Europe centrale et orientale, pose la question des rapports existant entre des mouvements simultanés mais dispersés géographiquement et cherche à comprendre la manière dont non seulement les mouvements sociaux mais aussi les contextes politiques sont entrés en résonance et ont finit par former un réseau d'événements qui n'est réductible ni à une série d'actions parallèles ayant subi des influences identiques, ni à une propagation du savoir par les médias. Afin de répondre à la question, il commence par un examen des problématiques disponibles en matière d'action collective montre leurs limites actuelles et propose quelques hypothèses élargissant la pertinence de la notion de cycle de contestation, et visant à rendre les concepts encore plus interactifs et encore plus historiques qu'auparavant.

Charles Tilly, basing himself on the 1989 events in central and eastern Europe, examines the relations between simultaneous, but geographically remote, social movements. He seeks an understanding of how such movements and political change achieve a " resonance " that gradually produces a network of events. These cannot be simply reduced to a series of parallel actions emanating from similar causes, nor to the power of the media. The author examines the insufficiency of current theory on collective action, and proposes hypotheses widening the concept of contestation cycles, emphasising interaction and historical factors.

\section{INDEX}

Mots-clés : démocratie, mobilisation, mouvements politiques, résistances politiques, sociologie historique

Index géographique : Europe centrale et orientale

Index chronologique : 1989

\section{AUTEUR}

\section{CHARLES TILLY}

Professeur d'histoire et de sociologie, directeur du Center for Studies and Social Change New School for Social Research de New York. 